\title{
The Ongoing COVID-19 Epidemic Curves Indicate Initial Point Spread in China with Log-Normal Distribution of New Cases Per Day with a Predictable Last Date of the Outbreak
}

\section{Stefan Olsson ${ }^{1,2^{*}}$}

${ }^{1}$ State Key Laboratory of Ecological Pest Control for Fujian and Taiwan Crops, College of Plant Protection

${ }^{2}$ Plant Immunity Center, Haixia Institute of Science and Technology, College of Life Science

Fujian Agriculture and Forestry University, No.15 Shangxiadian Road, Cangshan District, Fuzhou City, Fujian Province, China. P.C. 350002

* E-mail stefan@olssonstefan.com or stefan.olsson@fafu.edu.cn

\begin{abstract}
During an epidemic outbreak it is useful for planners and responsible authorities to be able to plan ahead to estimate when an outbreak of an epidemic is likely to ease and when the last case can be predicted in their area of responsibility. Theoretically this could be done for a point source epidemic using epidemic curve forecasting. The extensive data now coming out of China makes it possible to test if this can be done using MS Excel a standard spreadsheet program available to most offices. The available data is divided up for whole China and the different provinces. This and the high number of cases makes the analysis possible. Data for new confirmed infections for Hubei, Hubei outside Wuhan, China excluding Hubei as well as Zhejiang and Fujian provinces all follow a log-normal distribution that can be used to make a rough estimate for the date of the last new confirmed cases in respective areas.
\end{abstract}

\section{Introduction}

In epidemics starting as a point source the number of new cases often follows a log-normal distribution or more precisely a Poisson-Gamma distribution. How this distribution will develop over time can theoretically be determined by fitting a log-normal distribution equation to the data for new cases per day are reported. The estimate will of cause be more accurate the further into the outbreak. A literally "breaking point" for the accuracy of the estimate for the end of the outbreak comes after the number of new cases per day have reached its peak. From there on the estimate should be better and better. Here a simple method that could be used without access to special resources for getting such estimates after the peak has been reached is presented using data from the ongoing COVID-19 epidemic in China.

\section{Results and discussion}

A log normal distribution can be relatively nicely fitted all data sets (Fig 1\&2). When using a log scale for the $\mathrm{Y}$-axis it is apparent there are deviations in the early dates especially for Hubei (Fig $1 \mathrm{~A})$. This could be caused by a lag in detection of new cases in the beginning of the outbreak. The deviations in the latest dates can have many different causes like changing criteria for new cases, or simply a backlog in cases confirmation due to highly stressed health care system in the worst hit city Wuhan. Both the data from Hubei outside Wuhan (Fig 1B) and China outside Hubei (Fig 1C) on the other hand closely follows a log normal distribution. To see if the same relationships holds also outside Hubei, two provinces with quite different number of cases, Zhejiang with many cases and Fujian with few cases, was also tested (Fig 2). 

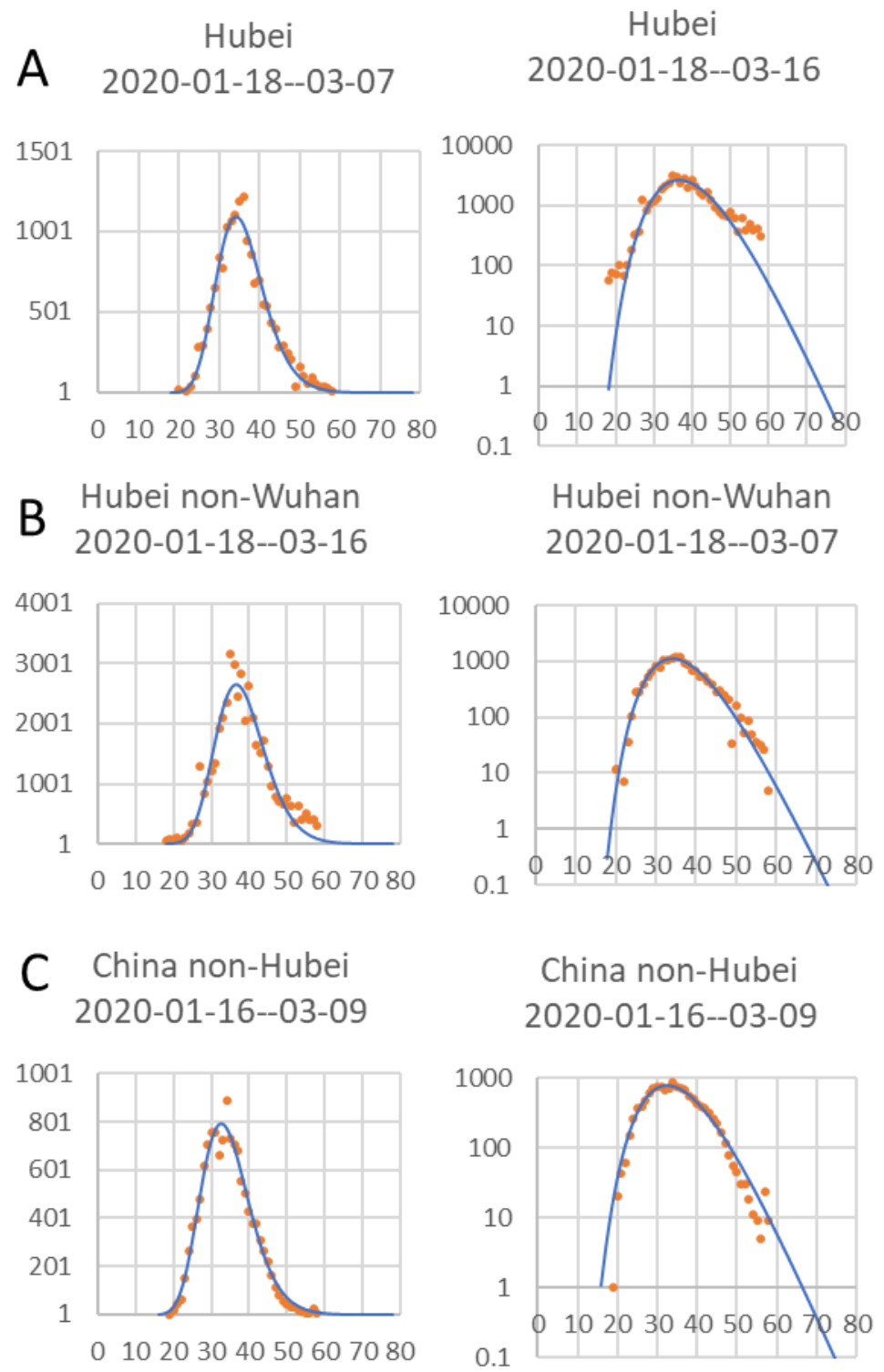

Figure 1. Log normal distribution of new confirmed cases for each day since 1 Jan 2020 Hubei, Hubei-nonWuhan and in reest of China. The Log of day values with start on the first day a case could have been confirmed was used curve fitting although here in the plot the actual number of days since $1^{\text {st }}$ January was used as $\mathrm{X}$-axis. Number of new confirmed cases per day and fitted curve (left) and Log number of new cases per day to show start and stop days (right). Headings shows estimated dates for $1^{\text {st }}$ and last confirmed case. $Y$ axes both to the left and right start at 1 to highlight the first and last case.

In Zheijiang the outbreak followed the general pattern very closely (Fig 2A) but for the much smaller outbreak in Fujian (Fig 2B) the number of cases per day dropped more than the model for the last days. This is caused by the approximation to log-normal distribution instead of a Poisson distribution that is more correct for data with few cases (Gonzales-Barron and Butler, 2011) but more difficult to handle 
using standard Excel curve fitting. This discrepancy mean that the last new infection date will be overestimated especially for limited outbreakes like the one in Fujian province. From planning point of view it should however be safer to oversestimate the length of the outbreak than underestimate it. A fairly good estimate of the last data could be done as soon as the number of new confirmed cases per day started to decrease.

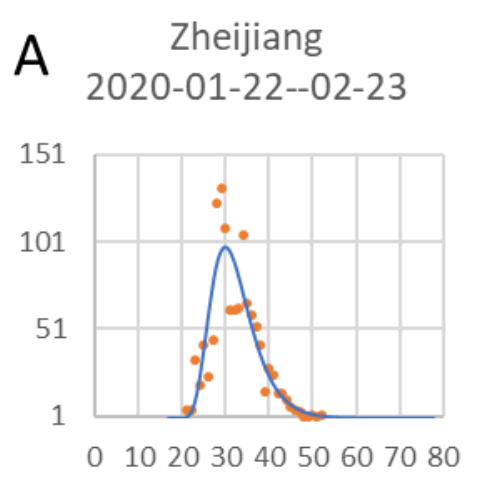

Fujian
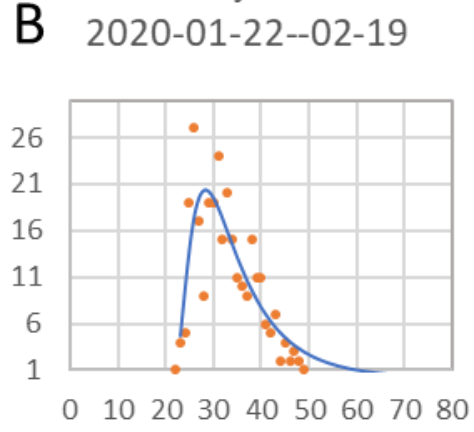

Zheijiang 2020-01-22--02-23

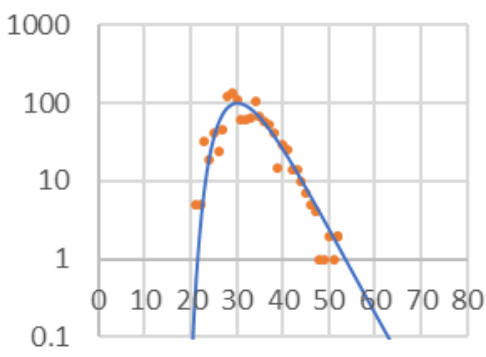

Fujian

2020-01-22--02-19

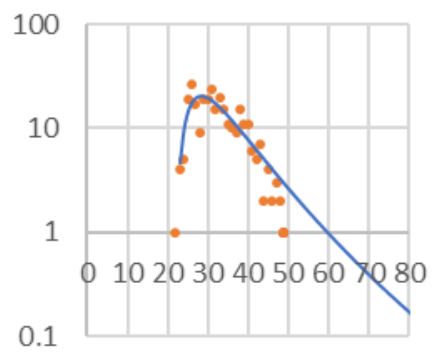

Figure 2. Log normal distribution of new confirmed cases for each day since 1 Jan 2020 in two provinces with relatively high numers of cases, Zeijiang with high numbers and Fujian with low numbers. The Log of day values with start on the first day a case could have been confirmed was used curve fitting although here in the plot the actual number of days since $1^{\text {st }}$ January was used as X-axis. Number of new confirmed cases per day and fitted curve (left) and Log number of new cases per day to show start and stop days (right). Headings shows estimated dates for $1^{\text {st }}$ and last confirmed case. Y-axes both to the left and right start at 1 to highlight the first and last case.

The estimated start date for when new cases could have been confirmed caused by community spread was for Hubei and Wuhan the $18^{\text {th }}$ January while outside Hubei the data indicate a 2 day earlier start if the disease behaved similarly. This is a bit surprising but could indicate that the disease was brought to Wuhan city and Hubei province from a less populated area and found good conditions for spread in Wuhan. The estimated start dates for when new cases could be confirmed in the two provinces Zheijiang and Fujian were both the $22^{\text {nd }}$ January a few days later than in the epicenter for the outbreak.

\section{Conclusion}


information should be useful to people in charge for planning how to allocate resources. The information will also be available when resources are as most stretched with a large number of active cases just after the peak in number of new cases per day, In addition, if the data continue to fit the curve for a point source outbreak in one area there has most likely been no new introduction of cases or any change to the virus or the likelihood that a person becomes infected within that area. The latter seems to be the case for the COVID-19 outbreak in China 2019-2020 pointing to that the quarantining measures stopping further spread between provinces and cities after the first few days of person-toperson transfer have worked efficiently.

\section{Methods}

Official referred to data for the COVID-19 outbreak in China is collected at a Wikipedia page (Anonymous, 2020). Since the kind of analysis here presented is a relatively simple analysis it should be possible to do for anyone using a standard program Microsoft Excel with the standard available Solver plugin for data handling and curve fitting. The logarithm of number of days since the estimated start of the epidemic outbreak were used for fitting a normal distribution equation to the data but in the figures the data was plotted against the non-logged day number with day 1 on the $1^{\text {st }}$ January to ease in determining the actual dates from readings on the $\mathrm{X}$-axis and the values in the spreadsheet files.

The MS Excel file used for this analysis is available as Supplementary file and can easily be modified to be used with other data to relatively early after the peak in new confirmed cases be able to predict the end of an epidemic outbreak with a definite starting point having a "first case".

\section{Acknowledgement}

When back in my home country Sweden I had to decide when to return to China after the winter break for the Chinese New Year (Spring Festival), I decided to look at the epidemiology data since I have been working with biological control trying to cause epidemics in fungal pathogens attacking plants. I thought of looking for data about the COVID-19 outbreak to be able to determine a time and a route back that limit the chances for me to catch the infection and bring it to my workplace. I found the very good Wikipedia entry I refer to in the methods and would like to thank everyone that has contributed to edit that site. Finally, I want to acknowledge my employer Fujian Agriculture and Forestry University that makes it possible for me to do research in China.

\section{Supplemental file}

"Corona model final.xlsx" is a supplemental file containing all data and calculations. In addition, the file also contain instructions for how to use it to fit new data to make predictions.

\section{References}

Anonymous (2020). Timeline of the 2019-20 coronavirus outbreak. WikipediA. Available at: https://en.wikipedia.org/wiki/Timeline_of_the_2019\%E2\%80\%9320_coronavirus_outbreak\#Cas e_statistics [Accessed March 1, 2020]. 
112 Gonzales-Barron, U., and Butler, F. (2011). A comparison between the discrete Poisson-gamma and 113 Poisson-lognormal distributions to characterise microbial counts in foods. Food Control 22, 114 1279-1286. doi:10.1016/j.foodcont.2011.01.029.

115 